\title{
Ueber die Wendungsberührebenen der Raumcurven.
}

(Von Herrn Joh. Nik. Bischoff zu München.)

W/s ist im $56^{\text {sten }}$ Bande dieses Journals p. 177 bemerkt worden, dafs einer Raumcurve $m n^{\text {ter }}$ Ordnung $2 m n(3 m+3 n-10)$ Wendungsberührebenen, d. i. solche Ebenen zukommen, welche mit ihr vier auf einander folgende Punkte gemein haben. Man kann dies unmittelbar in folgender Weise zeigen. Es seien

(1.) $f=0$ und $\varphi=0$

die Gleichungen der Raumcurve und $f$ und $\varphi$ vom $m^{\text {ten }}$ und $n^{\text {ten }}$ Grade. Für die Schmiegungsebene im Punkte $(x, y, z)$ der Curve (1.) hat man nach Herrn Hesse $\left(41^{\text {ster }} \mathrm{Bd} .\right.$, p. 283):

$$
\left(\xi \varphi_{1}+\eta \varphi_{2}+\zeta \varphi_{3}+\sigma \varphi_{4}\right) \frac{P}{(m-1)^{2}}-\left(\xi f_{1}+\eta f_{2}+\zeta f_{3}+\sigma f_{4}\right) \frac{Q}{(n-1)^{2}}=0
$$

wo $\boldsymbol{P}$ und $\boldsymbol{Q}$ homogene Functionen vom $(3 m+2 n-8)^{\text {ten }}$ und $(3 n+2 m-8)^{\text {ten }}$ Grade der Coordinaten $(x, y, z, s)$ sind.

Wählt man auf der Curve (1.) einen beliebigen Punkt $\left(x_{1}, y_{1}, z_{1}\right)$, bezeichnet die ihm entsprechenden Werthe von $f_{1}, f_{2}$, etc., $\varphi_{1}, \varphi_{2}$, etc., $\boldsymbol{P}$ und $\boldsymbol{Q}$ durch $f_{1}^{\prime}, f_{2}^{\prime}$, etc., $\varphi_{1}^{\prime}, \varphi_{2}^{\prime}$, etc., $\boldsymbol{P}^{\prime}$ und $\boldsymbol{Q}^{\prime}$ und nimmt man den Punkt $(\xi, \eta, \zeta)$ so, dafs

$$
\frac{\xi f_{1}^{\prime}+\eta f_{2}^{\prime}+\zeta f_{3}^{\prime}+\sigma f_{4}^{\prime}}{\xi \varphi_{1}^{\prime}+\eta \varphi_{2}^{\prime}+\zeta \varphi_{3}^{\prime}+\sigma \varphi_{4}^{\prime}}=\frac{\boldsymbol{P}^{\prime}}{\boldsymbol{Q}^{\prime}} \cdot \frac{(n-1)^{2}}{(m-1)^{2}}=\lambda,
$$

dann hat von den Schmiegungsebenen, die durch den Punkt $(\xi, \eta, \zeta)$ gehen, allemal eine ihren Schmiegungspunkt in $\left(x_{1}, y_{1}, z_{1}\right)$. Bestimmt man nun die letzteren Coordinaten oder $\lambda$ so, dafs die Fläche

$$
\frac{P}{(m-1)^{2}}-\lambda \frac{Q}{(n-1)^{2}}=0
$$

im Punkte $\left(x_{1}, y_{1}, z_{1}\right)$ die Curve (1.) berührt, so fallen von den Schmiegungsebenen des Punktes $(\xi, \eta, \zeta)$ zwei zusammen, es wird also eine von diesen Ebenen Wendungsberührebene.

Die Bedingungen für das Berühren von (1.) und (3.) im Punkte $\left(x_{1}, y_{1}, z_{1}\right)$ sind aber:

$$
f^{\prime}=0, \quad \varphi^{\prime}=0, \quad\left|\begin{array}{llll}
f_{1}^{\prime} & f_{2}^{\prime} & f_{3}^{\prime} & f_{4}^{\prime} \\
\varphi_{1}^{\prime} & \varphi_{2}^{\prime} & \varphi_{3}^{\prime} & \varphi_{4}^{\prime} \\
\boldsymbol{P}_{1}^{\prime} & \boldsymbol{P}_{2}^{\prime} & \boldsymbol{P}_{3}^{\prime} & \boldsymbol{P}_{4}^{\prime} \\
\boldsymbol{Q}_{1}^{\prime} & \boldsymbol{Q}_{2}^{\prime} & \boldsymbol{Q}_{3}^{\prime} & \boldsymbol{Q}_{4}^{\prime}
\end{array}\right|=0 .
$$

Also ergiebt sich als Anzahl der Wendungsberührebenen: $2 m n(3 m+3 n-10)$, und man sieht zugleich, dafs die Schmiegungspunkte dieser Ebenen einer Fläche von der $2(3 m+3 n-10)^{\text {ten }}$ Ordnung angehören.

Demnach lassen sich zwei Flächen zweiter Ordnung auf 16 Arten mit 
einer Ebene so schneiden, dafs die sich ergebenden Kegelschnitte eine vierpunktige Berührung haben.

Ist die Curve (1.) Durchschnitt zweier Cylinderflächen $\boldsymbol{m}^{\text {ter }}$ und $\boldsymbol{n}^{\text {ter }}$ Ordnung, so reducirt sich die vorhergehende Anzahl auf : $m n(5 m+5 n-18)$. Da die abwickelbare Fläche $W$, welche die Curve (1.) zur Rückkehrlinie hat, von der Ordnung $m \boldsymbol{n}(\boldsymbol{n}+\boldsymbol{n}-2)$ ist, so steigt die Bedingungsgleichung (a) für das Berühren einer Ebene und der Curve (1.) hinsichtlich der Coefficienten der Ebene auf den Grad $m n(m+n-2)$. Denkt man sich nun die Coefficienten der Schmiegungsebene (2.) eingeführt in die Bedingung $(a)$, so erhält man eine Gleichung $(b)$ vom $3 m n(m+n-2)(m+n-3)^{\text {ten }}$ Grade in $x, y, z$. Diese Gleichung $(b)$ zerfällt aber offenbar 1 ) in die Gleichung der abwickelbaren Fläche $W, 2$ ) in die Gleichung derjenigen Fläche, deren Schnittpunkten mit der Curve (1.) Schmiegungsebenen zukommen, von welchen diese Curve noch in einem andern Punkte berührt wird und endlich 3) in die Gleichung derjenigen Fläche, welche die Curve (1.) in ihren Wendeschmiegungspunkten schneidet. Jeder Wendeschmiegungspunkt kann aber viermal als solcher Punkt genommen werden, dessen Schmiegungsebene die Curve (1.) noch in einem andern Punkte berührt. Heifst also $t$ die Anzahl der Schmiegungsebenen, von welchen die Curve (1.) aufser im Schmiëgungspunkt noch anderswo berührt wird, so hat man: $t=m n(3 m+3 n-10)\{m n(m+n-2)-8\}$. Die Curve (1.) hat unter ihren Tangenten auch solche, von welchen sie aufser im Berührpunkt noch anderswo geschnitten wird. Man findet die Anzahl $v$ dieser Tangenten durch das Verfahren, welches Jacobi für die Bestimmung der Anzahl der Doppeltangenten ebener algebraischer Curven gegeben hat, und zwar folgt: $v=m n\{(m-2)(m n+n-4)+(n-2)(m n+m-4)\}$. Es liegen also die Berührpunkte dieser Tangenten zugleich in einer Fläche von der Ordnung: $(m-2)(m n+n-4)+(n-2)(m n+m-4)$.

Wählt man die Curve (1.) als Durchschnitt eines einfachen Hyperboloides und einer Fläche $\boldsymbol{n}^{\text {ter }}$ Ordnung, so giebt die vorhergehende Formel:

$$
v=4 m(m-1)(m-2) \text {. }
$$

Bemerkt man, dafs jeder Strahl des Hyperboloides, welcher die Pläche $m^{\text {ter }}$ Ordnung berührt, (m-2) Tangenten der Schnittcurve vertritt, von welchen diese noch anderswo getroffen wird, so erhält man als Anzahl der die Fläche $m^{\text {ter }}$ Ordnung berührenden Strahlen: $4 m(m-1)$, was sich auch auf anderem Wege leicht als richtig nachweisen lậfst.

München, im Februar 1860. 